\title{
ON THE GEOMETRIC THEORY OF FUNCTIONS MEROMORPHIC IN A DISC'1
}

\author{
BY J. E. MCMILLAN
}

Communicated by Maurice Heins, July 25, 1967

Let $w=f(z)$ be a nonconstant meromorphic function defined in the open unit disc $D$, and let $W$ denote the extended w-plane. Let $N(w, \delta)$ denote the set of all points of $W$ at a chordal distance less than $\delta$ from $w(\delta>0)$, and define a closed set $B C W$ as follows: $w \in B$ if and only if $w \in W$ and for any $\delta>0$ there exist $N\left(w_{0}, \delta_{0}\right)$ $C N(w, \delta)$ and a component $U$ of the preimage $f^{-1}\left(N\left(w_{0}, \delta_{0}\right)\right)$ such that $f(U)$ is not dense in $N\left(w_{0}, \delta_{0}\right)$.

THEOREM 1. Suppose that $V$ is a domain contained in the complement of $B$ and that $U$ is a component of $f^{-1}(V)$. Then one of the following two statements holds:

(i) For any $w \in V$ there exists $\delta>0$ such that $U \cap f^{-1}(N(w, \delta))$ is relatively compact (in $D$ ).

(ii) For any $w \in V$ either there exists a continuous curve $\alpha: z(t)$, $0 \leqq t<1$, lying in $U$ such that $|z(t)| \rightarrow 1$ and $f(z(t)) \rightarrow w$ as $t \rightarrow 1$, or there exists $\delta>0$ such that infinitely many relatively compact components of $f^{-1}(N(w, \delta))$ are contained in $U$.

The proofs of the results stated in this note will appear in a forthcoming paper.

A point $w \in W$ is called an asymptotic value of $f$ provided there exists a continuous curve $\alpha: z(t), 0 \leqq t<1$, lying in $D$ such that $|z(t)| \rightarrow 1$ and $f(z(t)) \rightarrow w$ as $t \rightarrow 1$, and if in addition $z(t) \rightarrow e^{i \theta}$, then $f$ is said to have the asymptotic value $w$ at $e^{i \theta}$. Define a set $\Gamma_{p} \subset W$ as follows: $w \in \Gamma_{p}$ if and only if $w \in W$ and there exists $e^{i \theta}$ such that $f$ has the asymptotic value $w$ at $e^{i \theta}$. For any set $S \subset W$, let $A(S)=$ $\left\{e^{i \theta}\right.$ : there exists $w \in S$ such that $f$ has the asymptotic value $w$ at $\left.e^{i \theta}\right\}$.

Theorem 2. Suppose that $w \in B$. Then for any $\delta>0, A(N(w, \delta))$ has positive Lebesgue measure (in $[0,2 \pi]$ ) and $\Gamma_{p} \cap N(w, \delta)$ has positive linear measure.

We use the definition of linear measure that is given in terms of coverings by discs. The measurability of $A(N(w, \delta))$ and $\Gamma_{p}$ is proved in (7).

\footnotetext{
${ }^{1}$ Supported partially by National Science Foundation grant GP-6538.
} 
Theorem 3. Suppose that $V$ is a component of the complement of $B$. Then one of the following two statements holds:

(1) There are at most finitely many components $U$ of $f^{-1}(V)$, each of which satisfies (i). In particular, $f$ assumes as a value every point of $V$ the same finite number of times $n$, multiplicities counted as usual (possibly $n=0$ ).

(2) For any $w \in V$, either $w$ is an asymptotic value of $f$, or the following holds: There exists $\delta>0$ such that $f^{-1}(N(w, \delta))$ has infinitely many relatively compact components, and in particular $f$ assumes as a value every point of $N(w, \delta)$ infinitely many times.

Moreover, if in case (2) we let $n=+\infty$ (in this case each point of a residual subset of $V$ is assumed by $f$ infinitely many times), then any (curvilinearly) accessible boundary point of $V$ that is assumed by $f$ less than $n$ times $(1 \leqq n \leqq+\infty)$, multiplicities counted as usual, is an asymptotic value of $f$.

Let $\Gamma$ denote the set of all asymptotic values of $f$. The global cluster set $C$ and the range of values $R$ are defined as follows: $w \in C$ if and only if $w \in W$ and there exists a sequence $\left\{z_{n}\right\} \subset D$ such that $\left|z_{n}\right| \rightarrow 1$ and $f\left(z_{n}\right) \rightarrow w$. $w \in R$ if and only if $w \in W$ and there exists a sequence $\left\{z_{n}\right\} \subset D$ such that $\left|z_{n}\right| \rightarrow 1$ and $f\left(z_{n}\right)=w$. Let int $R$ denote the interior of $R$. We also define $F$ to be the set of all $w \in W$ that satisfy the conclusion of Theorem 2. Then $F$ is closed and by Theorem $2, B C F$. Thus as a consequence of Theorem 3 we have the following result.

Corollary. $C-F$ is open, and the following two equivalent inclusions hold:

$$
C-F \subset \Gamma \cup \text { int } R ; \quad C-\text { int } R \subset \Gamma \cup F \text {. }
$$

Moreover, any accessible boundary point of $C-F$ that is not in $R$ is in $\Gamma$.

Let $V$ denote a domain contained in $W$, and suppose that $U$ is a component of $f^{-1}(V)$. Define sets $R(U)$ and $F_{c}$ as follows: $w \in R(U)$ if and only if $w \in V$ and $U \cap f^{-1}(\{w\})$ is infinite. $w \in F_{c}$ if and only if $w \in W$ and for any $\delta>0, A(N(w, \delta))$ has positive Lebesgue measure (in $[0,2 \pi]$ ) and $\Gamma_{p} \cap N(w, \delta)$ contains a closed set of positive (logarithmic) capacity. Then $F_{c}$ is closed and $B \subset F C F_{c}$.

THEOREM 4. If $V \cap B=\varnothing$, then either (i) holds or $V-R(U)$ contains no (nondegenerate) continuum. If $V \cap F=\varnothing$, then either (i) holds or $V-R(U)$ has linear measure zero. If $V \cap F_{c}=\varnothing$, then either (i) holds or $V-R(U)$ contains no closed set of positive capacity. 
We conclude with a discussion of the relationship between our results and some earlier theorems. The inclusion $C$-int $R \subset \Gamma \cup F$ refines the inclusion $C$-int $R \subset \bar{\Gamma}$ given by Collingwood and Cartwright [2]. It improves their result $W-R \subset \Gamma$ under the assumption that $\Gamma$ has linear measure zero, for we see that under the weaker assumption $F=\varnothing$, we have the stronger conclusion $W$-int $R \subset \Gamma$ (since $C-F$ is open and $C \neq \varnothing, C=W$ if $F=\varnothing$ ). If $f$ is bounded and has radial limits of modulus one at almost all $e^{i \theta}$, then it is an immediate consequence of Theorems 2 and 3 that $B=F=\{|w|=1\}$, and we see that the corollary sharpens a theorem of Seidel [9] (see also Frostman [3]). Let us now consider the less restrictive case where $f$ is only assumed to have an asymptotic value at each point of a set on $\{|z|=1\}$ of measure $2 \pi$. Let $E$ be a closed subset of $W$ such that for almost all $e^{i \theta}, f$ has an asymptotic value at $e^{i \theta}$ that is in $E$. By Bagemihl's ambiguous-point theorem [1], $F \subset E$ (also $F_{c} \subset E$ ), and we see that our results contain some theorems of Lehto [4] and Lohwater [5]. Our results also contain theorems of Noshiro [8], Stoillow [10], and McMillan [6].

\section{REFERENCES}

1. F. Bagemihl, Curvilinear cluster sets of arbitrary functions, Proc. Nat. Acad. Sci. U.S.A. 41 (1955), 379-382.

2. E. F. Collingwood and M. L. Cartwright, Boundary theorems for a function meromorphic in the unit circle, Acta Math. 87 (1952), 83-146.

3. O. Frostman, Potentiel d'equilibre et capacité des ensembles, Thèse, Lund, 1935.

4. O. Lehto, Value distribution and boundary behavior of a function of bounded characteristic and the Riemann surface of its inverse function, Ann. Acad. Sci. Fenn. Ser. A I 177 (1954), 1-46.

5. A. J. Lohwater, The boundary values of a class of meromorphic functions, Duke Math. J. 19 (1952), 243-252.

6. J. E. McMlllan, Asymptotic values of functions holomorphic in the unit disc, Michigan Math. J. 12 (1965), 141-154.

7. - On local asymptotic properties, the asymptotic value sets, and ambiguous properties of functions meromorphic in the open unit disc, Ann. Acad. Sci. Fenn. Ser. A I 384 (1965), 1-12.

8. K. Noshiro, Contributions to the theory of singularities of analytic functions, Japan. J. Math. 19 (1948), 299-327.

9. W. Seidel, On the distribution of values of bounded analytic functions, Trans. Amer. Math. Soc. 36 (1934), 201-226.

10. S. Stoillow, Les proprittes topologiques des fonctions analytiques d'une variable, Ann. Inst. H. Poincaré Sect. A 2 (1932), 233-266.

UNIVERSITY OF WisCONSIN-MILWAUKEE 